\title{
Modified model of droplet's spreading on solid body in presence of liquid microfilm
}

\author{
Zbigniew Zapałowicz \\ West Pomeranian University of Technology, Department of Heat Engineering, al. Piastów 17, 71-296 Szczecin, \\ Poland
}

\begin{abstract}
The paper presents a modified model of the phenomenon of droplet's spreading on a surface, in presence of a thin liquid microfilm. The presence of the thin microfilm affects the size of maximal droplet's spreading area. Presented model allows to calculate the maximal droplet's spreading radius, if parameters of the thin liquid film, as well as the macroscopic static Young angle are known.
\end{abstract}

\section{Introduction}

The process of wetting the surface of a solid body by a liquid in presence of a gas phase is to be observed in nature, as well as in numerous processes in technology [1]. Liquid can be supplied onto the surface of a solid body in the form of droplets or streams. A single droplet spreads after its contact with the surface. The spreading process is a very compound phenomenon and numerous parameters characterizing the system droplet-surfaceenvironment decide about its run. Significant parameters of the liquid are: kind of liquid which is decisive for its physical properties, and droplet's temperature and velocity in the moment of its contact with the surface. Kind of material, impurities and roughness of the surface, its shape and inclination are significant factors that characterize a solid body [2]. In turn, gas phase that surrounds the surface can be decisive for the formation of

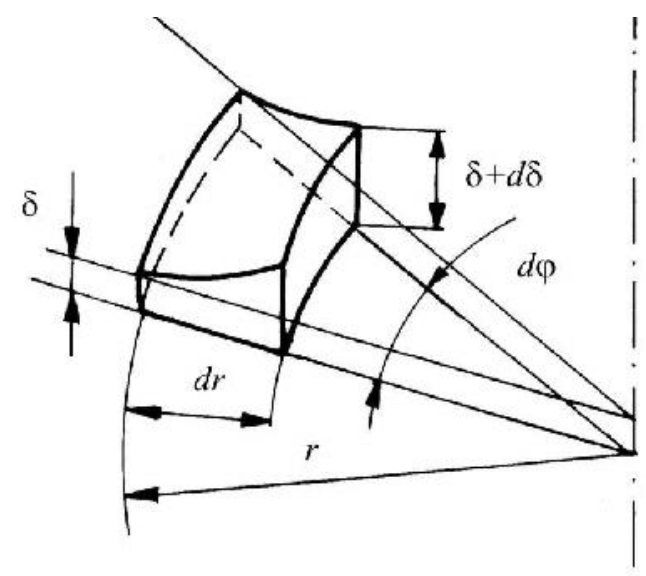

Figure 1. Control volume of droplet.

Corresponding author: zbigniew.zapalowicz@zut.edu.pl a thin microfilm on the surface of the solid body [3-7]. The microfilm has influence on the droplet's spreading process. The spreading process of the droplet can be considered both in macroscopic and in microscopic ways [8-11].

The aim of the present paper is to elaborate such a model of the droplet's spreading process, that takes into account the effect of presence of a thin microfilm on the surface. The model describes droplet's behavior in the phase of its maximal spreading on the surface. In the moment of droplet's maximal spreading, there occurs equilibrium among liquid, solid body, and gas.

Presented model is a modification of a formerly elaborated model for the phenomenon presented a.o. in papers $[1,12,13]$.

\section{Model}

Problems to verify the model presented in papers [14, 15], of droplet's spreading phenomenon in presence of a microfilm, have triggered further investigations. The present paper shows the modified mathematical description of the mode of relations between the liquid and the surface of a solid body. In the moment when the process of droplet's spreading comes to a stand-still, forces affecting droplet in radial direction come to an equilibrium state.

Assumptions made in papers $[1,12,13]$ remain valid, and they can be given as follows:

- surface is horizontal, smooth, heterogenic and free from micro-impurities,

- surface is coated with a thin liquid microfilm on which the liquid spreads,

- there is a lack of thermal relations among surface, droplet and environment; the liquid's spreading process is isothermal, 
- liquid is homogeneous,

- as a result of intermolecular forces in interfacial liquid-gas region in the liquid microfilm and in microscopic region, additional pressure forms, it is called disjoining pressure [6],

- it is assumed that Van der Waals forces affect dominantly the value of intermolecular relations $[4,5$, 7, 16-18].

Control volume has been separated from the droplet in the phase of its maximal spreading (Fig.1). External forces affect the control areas of the droplet.

Particular forces affecting the areas of control volume of the liquid in radial direction are described by equations given below:

- force directed from the liquid microfilm

$$
F_{1}=p_{l} \delta r d \varphi
$$

- force directed from the fundamental volume of droplet

$$
F_{2}=\left(p_{l}+d p_{l}\right)(\delta+d \delta)(r-d r) d \varphi
$$

- forces directed from the neighboring layers of liquid

$$
F_{3,4}=\left(p_{l}+\frac{d p_{l}}{2}\right)\left(\delta+\frac{d \delta}{2}\right) \sin \frac{d \varphi}{2} d r
$$

- affecting force of the gas

$$
F_{5}=p_{g}\left(r-\frac{d r}{2}\right) \frac{d r}{\cos \alpha} \sin \alpha d \varphi
$$

- force of adhesion

$$
F_{6}=\Delta \sigma d r d \varphi
$$

The last of given equations results from the analysis of the phenomenon of adhesion. Change of length of droplets wetting circumference by the following value:

$$
\Delta l=r d \varphi-(r-d r) d \varphi=d r d \varphi
$$

requires the effect of adhesion to be cleared away [19]

$$
\Delta \sigma=\sigma_{\mathrm{lg}}\left(1-\cos \theta_{Y}\right)
$$

In the moment when the droplet's spreading process comes to a stand-still, equilibrium of above given radial forces must occur, thus:

$$
-F_{1}+F_{2}+F_{3}+F_{4}-F_{5}-F_{6}=0
$$

After substitution of relations (1)-(7) in equation (8), the following relation was obtained:

$$
p_{l} \cdot r \frac{\mathrm{d} \delta}{\mathrm{d} r}+\delta \cdot r \frac{\mathrm{d} p_{l}}{\mathrm{~d} r}-p_{g} \cdot r \cdot \operatorname{tg} \alpha-\sigma_{\mathrm{lg}} \cdot\left(1-\cos \theta_{Y}\right)=0
$$

If only Van der Waals forces are significant, disjoining pressure can be introduced in the analysis, given by the following equation:

$$
p_{d}=p_{g}-p_{l}=\frac{A_{d}}{\delta^{3}}
$$

If $p_{g}=i d e m$ then, after differantation of equation the following equation was obtained:

$$
-\frac{d p_{l}}{d r}=-\frac{3 A_{d}}{\delta^{4}} \frac{d \delta}{d r}
$$

Assuming that

$$
\operatorname{tg} \alpha=\frac{d \delta}{d r}
$$

equation (9) can be written as:

$$
\frac{2 A_{\mathrm{d}}}{\sigma_{\mathrm{lg}} \cdot\left(1-\cos \theta_{Y}\right)} \frac{\mathrm{d} \delta}{\delta^{3}}=\frac{d r}{r} .
$$

By integrating on both sides equation (13), the following relation was obtained:

$$
\frac{-A_{d}}{\sigma_{\mathrm{lg}} \cdot\left(1-\cos \theta_{Y}\right)} \cdot \frac{1}{\delta^{2}}=\ln |r| .
$$

Hence:

$$
r=e^{\frac{-A_{d}}{\sigma_{\lg } \cdot\left(1-\cos \theta_{Y}\right)} \cdot \frac{1}{\delta^{2}}}
$$

For the borderline between the absorbed liquid microfilm region and contact region, the following condition is valid:

$$
r=R_{\max } \quad \delta=\delta_{0},
$$

and:

$$
R_{\max }=e^{\frac{-A_{d}}{\sigma_{\lg } \cdot\left(1-\cos \theta_{Y}\right)} \cdot \frac{1}{\delta_{0}^{2}}}
$$

Determination of maximal wetting radius requires determination of thickness of the liquid microfilm in the moment when droplet stops its movement and determination of macroscopic wetting angle (Young angle) of the surface on which the droplet rests. It is also necessary to know the dispersion constant for the system: droplet- material the surface was made of.

Thickness of adsorbed film can be estimated from model given by Ajaev [20]:

$$
\delta_{0}=\delta^{+} \cdot C a^{1 / 3} \cdot R_{0}
$$

Model of Ajaev is valid for evenly heated surface area. Dimensionless thickness of absorbed film is determined by relation:

$$
\delta^{+}=\left(\frac{\zeta^{+} \cdot \varepsilon^{+}}{T_{0}^{+}}\right)^{1 / 3}
$$

In equation (19) there are three dimensionless parameters. First one characterizes the effect of changes in liquid 
pressure on the local phase-change temperature at the interface:

$$
\zeta^{+}=\frac{\sigma_{\mathrm{lg}}}{b \cdot \rho \cdot R_{0} \cdot C a^{1 / 3}}
$$

Second one is the disjoining pressure parameter:

$$
\varepsilon^{+}=\frac{\left|A_{\text {Ham }}\right|}{\sigma_{\mathrm{lg}} \cdot R_{0}^{2} \cdot C a}
$$

and third one represents non-dimensional temperature:

$$
T_{0}^{+}=\frac{T_{0}-T_{n}}{\mathrm{Ca}^{2 / 3} \cdot T_{n}}
$$

Capillary number in equations (18) and (20-22) is defined as:

$$
C a=\frac{v \cdot \rho \cdot u}{\sigma_{\lg }}
$$

The characteristic velocity in (23) is given by equation:

$$
u=\frac{\lambda \cdot T_{n}}{\rho \cdot b \cdot R_{0}}
$$

The surface tension $\sigma_{\mathrm{lg}}$ is determined at the equilibrium saturation temperature $T_{n}$ and characteristic liquid's parameters $b, \lambda, v, \rho$ are given at temperature of liquid.

Relation between the Hamaker constant and the dispersion constant is described by the equation:

$$
A_{H}=6 \cdot \pi \cdot A_{d}
$$

\section{Calculations}

Calculations are made for water droplets on the surface of a glass plate. Hamaker constant for water droplets spreading on the surface of a glass plate given by Gregory [21] is equal $A_{\text {Ham }}=5,86 \cdot 10^{-20} \mathrm{~J}$. Thus the dispersion coefficient, according to (25), equals $A_{\mathrm{d}}=3,11 \cdot 10^{-21} \mathrm{~J}$.

Table 1. Physical parameters of water [22].

\begin{tabular}{|l|c|c|c|}
\hline \multicolumn{1}{|c|}{ Parameter } & Symbol & Unit & Value \\
\hline Temperature & $t_{n}$ & ${ }^{\circ} \mathrm{C}$ & 20 \\
\hline Density & $\rho$ & $\mathrm{kg} / \mathrm{m}^{3}$ & 998,2 \\
\hline Latent vaporization & $b$ & $\mathrm{~kJ} / \mathrm{kg}$ & 2453,8 \\
\hline Thermal conductivity & $\lambda$ & $\mathrm{W} /(\mathrm{mK})$ & 0,599 \\
\hline Kinematic viscosity & $v$ & $\mathrm{~m}^{2} / \mathrm{s}$ & $1,006 \cdot 10^{-6}$ \\
\hline Surface tension & $\sigma$ & $\mathrm{N} / \mathrm{m}$ & 0,07269 \\
\hline
\end{tabular}

It is assumed that spreading water droplet has temperature of $20^{\circ} \mathrm{C}$, and temperature glass surface equals $30^{\circ} \mathrm{C}$. Physical parameters of water are given in Table 1.

Next, all parameters necessary to determine the thickness of absorbed film can be related only to the radius [13] by substitution of values of physical parameters of water given in Tab.1 in equations (18) (24). Then, respectively:

- characteristic velocity (24),

$$
u=C_{1} \cdot R_{0}^{-1}
$$

- Capillary number (23),

$$
\mathrm{Ca}=C_{2} \cdot R_{0}^{-1}
$$

- parameter characterizes the effect of changes in liquid pressure on the local phase-change temperature at the interface (20),

$$
\zeta^{+}=C_{3} \cdot\left(R_{0}\right)^{-2 / 3}
$$

- non-dimensional parameter (21),

$$
\varepsilon^{+}=C_{4} \cdot R_{0}^{-1}
$$

- non - dimensional temperature (22),

$$
T_{0}^{+}=C_{5} \cdot R_{0}^{2 / 3}
$$

- non - dimensional thickness of water film (19),

$$
\delta^{+}=C_{6} \cdot\left(R_{0}\right)^{-7 / 9}
$$

- thickness of water film (18),

$$
\delta_{0}=C_{7} \cdot\left(R_{0}\right)^{-1 / 9}
$$

are only functions of radius $R_{0}$, related to droplet's volume $V_{0}$.

The radius of spherical droplet is the function of its volume:

$$
R_{0}=\left(\frac{3 \cdot V_{0}}{4 \cdot \pi}\right)^{1 / 3}
$$

Table 2. Characteristic coefficients for equations (26) - (32).

\begin{tabular}{|c|c|c|}
\hline Coefficient & Unit & Value \\
\hline$C_{1}$ & $\mathrm{~m}^{2} / \mathrm{s}$ & $7,1690 \cdot 10^{-8}$ \\
\hline$C_{2}$ & $\mathrm{~m}$ & $9,9038 \cdot 10^{-10}$ \\
\hline$C_{3}$ & $\mathrm{~m}^{2 / 3}$ & $2,9773 \cdot 10^{-8}$ \\
\hline$C_{4}$ & $\mathrm{~m}$ & $8,1400 \cdot 10^{-10}$ \\
\hline$C_{5}$ & $\mathrm{~m}^{3 / 2}$ & 33757 \\
\hline$C_{6}$ & $\mathrm{~m}^{7 / 9}$ & $8,9542 \cdot 10^{-8}$ \\
\hline$C_{7}$ & $\mathrm{~m}^{10 / 9}$ & $8,9254 \cdot 10^{-11}$ \\
\hline
\end{tabular}


Values of characteristic coefficients $C$ in equations (26) - (32) are given in Tab.2.

Fig. 2 presents relations between microfilm thickness and water droplet's volume for assumed values of parameters. As it results from the model of Ajaev, thickness of the water microfilm forming on the heated surface area diminishes with increase of droplet's volume.

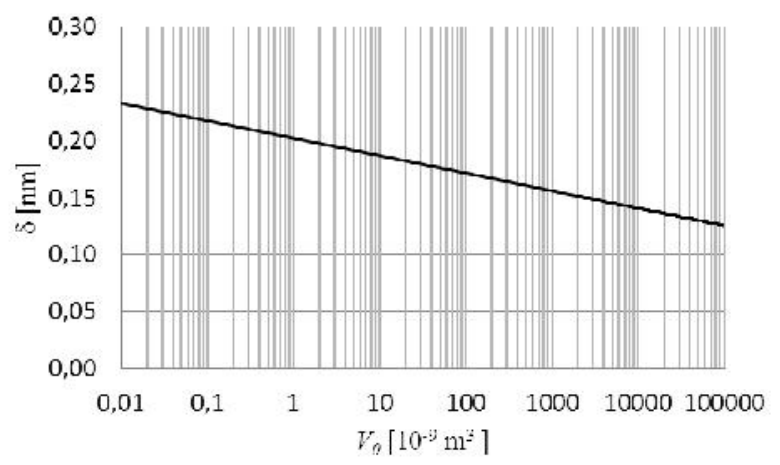

Figure 2. Relation of thickness of water microfilm to droplet's volume.

In turn, Fig. 3 shows results of calculations for water droplet's maximal spreading radius in relation to thickness of the liquid microfilm formed on the surface. It is assumed for calculations that equilibrium wetting angle (Young angle) equals $70^{\circ}$.

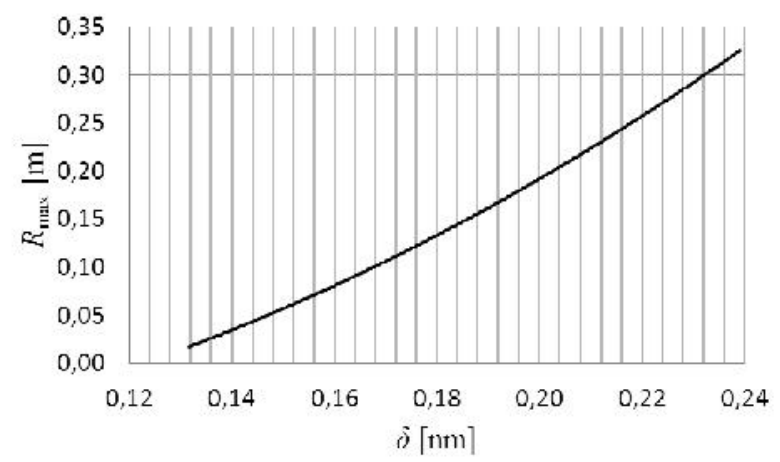

Figure 3. Relation of droplet's spreading radius to the value of liquid microfilm.

On the basis of Fig.3, it can be stated that with increase of water microfilm thickness, droplet's spreading area radius in the phase of droplet's maximal wetting area increases, too. However, values of droplet's spreading radius are too high in comparison with vales of this parameter obtained experimentally. One of the causative factors of the discrepancy can be imprecise estimation of adhesion force expressed in equation (6).

\section{Conclusions}

The paper presents the modified model of the process of liquid droplet's spreading on a flat, heated surface on which a thin liquid microfilm is present. The model makes it possible to calculate droplet's spreading area radius in the moment of droplet's maximal wetting area.
However, values of this radius are too high in relation to values obtained experimentally. One of possible causes of these discrepancies is imprecise determination of the adhesion force. Thus, the model requires further modifications.

\section{References}

1. Z. Zapałowicz, Effect of a droplet stream and air on the surface of a solid body (Technical University of Szczecin Publishers, 2007 (in Polish))

2. A. Marmur, Contact angle, wettability and adhesion, (Koninklijke Brill NV, 6, 3-18, 2009)

3. T.D. Blake, A. Clarke, J. De Coninck, J., M. Ruijter, M. Voue.. Colloids Surf. A., 149, 123-130, (1999)

4. K. Stephan, Int. J. Heat Mass Transfer, 45, 47154725, (2002)

5. P. Van Carey, Liquid-vapor phase-change Phenomena, (Bristol, Hemisphere Publishing Corporation, Taylor\&Francis, Pa,1992)

6. P.C. Wayner, Jr., Y.K. Kao, L.V. LaCroix, Int. J. Heat Mass Transfer, 19, 487-492, (1976)

7. P.C. Wayner, Jr., AIChE J., 45, 2055-2068, (1999)

8. E.D. Duszan, Ann. Rev. Fluid Mech., 371-400, 11, (1979)

9. P. Haley, M.J. Miksis, J. Fluid Mech., 223, 57-81, (1991)

10. S. Sciffer, Chem. Eng. Sci., 55, 5933-5936, (2000)

11. Z. Zapałowicz, Arch. Thermodynamics, 24, 55-68, (2003)

12. Z. Zapałowicz, 6th Int. Conf. HEAT 2011 (Ryn, Poland, 415-418, 2011)

13. Z. Zapałowicz, Arch. Thermodynamics. 33, 107-121, (2012)

14. Z. Zapałowicz Z., $14^{\text {th }}$ Int. Symp. HTRSE, (Międzyzdroje, Poland, 407-412, 2012)

15. Z. Zapałowicz, M. Sobczak, Analiza systemów energetycznych (Cracow, Poland, 361-372, 2013 (in Polish))

16. J.N. Israelachvilli, Intermolecular and surface forces, (Academic Press, London, 1991)

17. A. Majumdar, I. Mezic, J. Heat Transfer, 121, $965-$ 970 (1999)

18. E.K. Yeh, J. Newman, C.J. Radke, Colloids Surf., 156, 525-546 (1999)

19. R.E. Johnson Jr., R.H. Dettre, Surf. Colloid Sci., 2, 85-153 (1969)

20. V. Ajaev, J. Fluid Mech., 528, 279-296, (2005)

21. J. Gregory, Adv. Colloid Interface Sci., 2, 396-417, (1969)

22. Rivkin, S.L., Aleksandrov, A.A., Teplofizičeskie svojstva vody $i$ vodjanogo para, (Energia. 1980, (in Russian)) 